\title{
Mixed tocopherols are better than alpha-tocopherol as anti-oxidants- as good as statins
}

\author{
Zufeng Ding ${ }^{1,2}$, Tom GP Saldeen ${ }^{1,3}$, Pankaj Mathur, MD ${ }^{1}$, Jawahar L Mehta, MD, PhD ${ }^{1}$
}

Ding Z, Saldeen TGP, Mathur P, et al. Mixed tocopherols are better than alpha-tocopherol as anti-oxidant- as good as statins.
Curr Res Cardiol 2016;3(4):128-29.

$A$ therosclerosis is an inflammatory disease associated with oxidant Astress manifested as excess formation of reactive oxygen species (ROS) and high levels of oxidized low-density lipoprotein (ox-LDL) (1-3). Several early epidemiologic studies showed that routine use of vitamin E (as $\alpha$-tocopherol) resulted in a significant reduction in atherosclerosis-related events. However, subsequent randomized trials of $\alpha$-tocopherol failed to show any benefit of this therapy (4). It was suggested (5) that $\alpha$-tocopherol has limited anti-oxidant effect and other constituents of vitamin E, especially $\gamma$-tocopherol, might be necessary for the full benefits of vitamin E. Indeed, we demonstrated potent inhibitory effects of $\mathrm{y}$-tocopherol, more than $\alpha$-tocopherol, on platelet aggregation and in vivo thrombus formation (6).

Here we describe effects of $\alpha$ - and $\gamma$ - on tocopherol on arterial smooth muscle cells (SMCs)). These cells under the influence of ox-LDL transform into foam cells, and undergo proliferation and migration, express proinflammatory adhesion molecules and develop apoptosis- all features of atherosclerotic plaque. Ox-LDL binds to surface receptors, including the lectin-like ox-LDL receptor-1 (LOX-1) in SMCs. LOX-1 activation results in ox-LDL internalization, ROS generation, NF-kB activation, and expression of vascular cell adhesion molecule (VCAM-1) and pro-apoptotic genes (5). Of note, statins have been shown to modify ox-LDL/LOX-1-mediated events (7).

Human primary aortic SMCs were purchased from ATCC (Manassas, VA) and maintained in vascular cell basal medium. SMCs were treated with tocopherols in the absence or presence of simvastatin and ox-LDL for 24 h. Simvastatin, $\alpha$-tocopherol, $\beta$-tocopherol, $\gamma$-tocopherol and $\sigma$-tocopherol were purchased from Sigma.

$\alpha$ - and $\gamma$-tocopherol inhibited ox-LDL induced LOX-1 expression in a dose-dependent manner, while $\beta$-tocopherol alone had no effect and $\delta$-tocopherol a modest effect (Figure 1A). Hence, we focused our studies on the effects of $\alpha$-, $\delta$-, and $\mathrm{y}$-tocopherol on LOX-1 expression and its downstream effects on arterial SMCs exposed to ox-LDL $(20 \mu \mathrm{g} / \mathrm{ml})$. Parallel sets of SMCs were treated with simvastatin $(10 \mu \mathrm{M}), \alpha$-tocopherol and $\mathrm{\gamma}$-tocopherol alone $(10 \mu \mathrm{M}$ each), or a combination of $\mathrm{\gamma}$-tocopherol, $\delta$-tocopherol and $\alpha$-tocopherol (total $10 \mu \mathrm{M}$; in 5:2:1 ratio). As shown in Figure 1B, simvastatin decreased ox-LDL induced LOX-1 expression. $\gamma$ and $\alpha$-tocopherols also decreased LOX-1 expression ( $\gamma->\alpha$-tocopherol). Most interestingly, the combination of tocopherols had a very potent effectalmost as much as simvastatin. Next, we studied ox-LDL uptake by flow cytometry (Figure 1C), and observed a very potent inhibitory effect of the combination of tocopherols -about $75 \%$, again similar to the effect of simvastatin.

Next, we studied the status of VCAM-1 expression and NF-kB p65 activation (Figure 2A). As expected, there was intense VCAM-1 expression and NF-kB p65 activation in SMCs treated with ox-LDL. Simvastatin markedly reduced NF-kB p65 activation and VCAM-1 expression. Both $\gamma$ - and $\alpha$-tocopherols decreased NF-kB p65 activation and VCAM-1 expression ( $\gamma$-tocopherol >
A

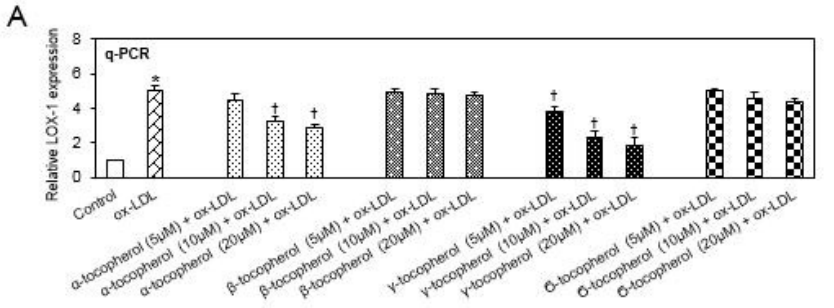

B

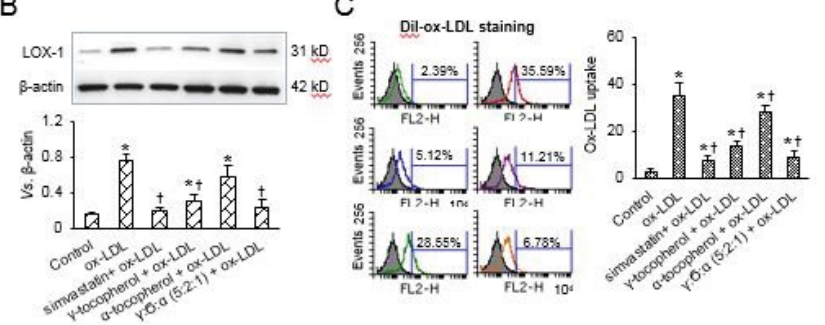

Figure 1) (A) $\alpha$-tocopherol and $\gamma$-tocopherol inhibited LOX-1 expression in a dose dependent manner, while $\beta$-tocopherol and $\sigma$-tocopherol almost have no effect on LOX-1 expression. In the presence of ox-LDL, pretreatment with simvastatin, $\alpha$-tocopherol and $\gamma$-tocopherol inhibited LOX-1 expression (B) and ox-LDL uptake (C). Bar graphs represent data in mean $\pm S D$ based on 5 independent experiments, ${ }^{*} P<0.05$ vs. control; $+P<0.05$ vs. ox $-L D L$

$\alpha$-tocopherol), and the tocopherols combination had a very potent inhibitory effect- almost as much as simvastatin. SMC apoptosis is associated with plaque rupture and promotion of outward remodeling as plaques resulting in aneurysm formation (1). Hence we investigated the effect of statins and tocopherols on SMC apoptosis. Caspase-3 (western blotting, Figure 2B) and TUNEL staining (flow cytometry, Figure 2C) showed that while both $\gamma$ - and $\alpha$-tocopherols decreased this marker of apoptosis $(\gamma$-tocopherol $>$ $\alpha$-tocopherol), the tocopherols combination had a very potent inhibitory effect-again as much as simvastatin, on SMC apoptosis (Figure 2C).

We believe this is the first study that examined the effect of different tocopherol components and their combination on ox-LL uptake, LOX-1 expression, adhesion molecule expression and markers of apoptosis.

$y$-tocopherol was absent from almost all preparation of vitamin $E$ used in clinical trials in the past. Its absence may be the basis for the negative results of vitamin $E$ trials. Based on the aforementioned studies, we suggest that $\mathrm{y}$-tocopherol should be a major component of vitamin E preparations for evaluation in anti-oxidant trials in primary or secondary prevention of cardiovascular diseases which are thought to be causally related to oxidative stress.

${ }^{1}$ Central Arkansas Veterans Healthcare System and the University of Arkansas for Medical Sciences, Little Rock, AR, USA, 2Key Laboratory for Biomechanics and Mechanobiology of Ministry of Education, School of Biological Science and Medical Engineering, Beihang University, China, ${ }^{3}$ Myeloma Institute, University of Arkansas for Medical Sciences, Little Rock, AR, USA

Correspondence: Dr. J.L. Mehta, MD, PhD, University of Arkansas for Medical Sciences, Little Rock, AR 72205, USA, Telephone 5019603102, e-mail MehtaJL@UAMS.edu

Received: September 13, 2016, Accepted: December 27, 2016, Published: December 29, 2016 


\section{Ding et al}

A

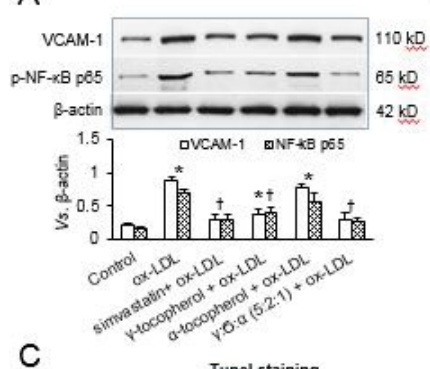

C

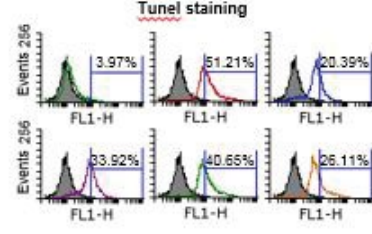

B
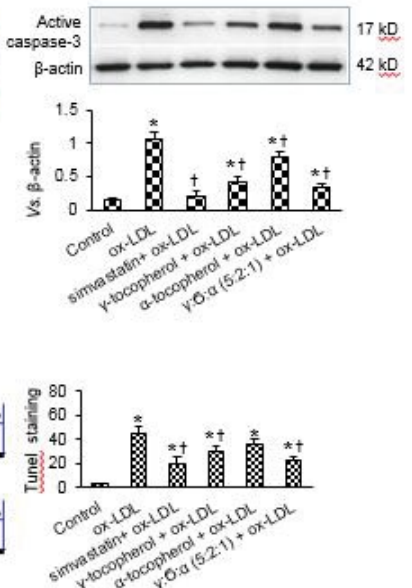

Figure 2) In the presence of ox-LDL, pretreatment with simvastatin, $\alpha$-tocopherol and $\gamma$-tocopherol inhibited expression of NF- $\kappa$ B and VCAM-1 (A), and apoptosis (B-active caspase-3, C- TUNEL staining). $\gamma$-tocopherol was more potent than $\alpha$-tocopherol and the tocopherol combination ( $\gamma: \delta$ : $\alpha$-tocopherols in 5:2:1 ratio) had the same effect as simvastatin. Bar graphs represent data in mean $\pm S D$ based on 5 independent experiments, ${ }^{*} P<0.05$ vs. control; $† P<0.05$ vs. ox-LDL

\section{REFERENCES}

1. Ding Z, Liu S, Wang X, et al. Oxidant stress in mitochondrial DNA damage, autophagy and inflammation in atherosclerosis. Sci Rep 2013;3:1077.

2. Ding Z, Liu S, Wang X, et al. LOX-1, mt-DNA damage, and NLRP3 inflammasome activation in macrophages: Implications in atherogenesis. Cardiovasc Res 2014;103:619-28.

3. Ding Z, Wang X, Schnackenberg L, et al. Regulation of autophagy and apoptosis in response to ox-LDL in vascular smooth muscle cells, and the modulatory effects of the microRNA hsa-let-7 g. Int J Cardiol 2013;168:1378-85.

4. Yusuf S, Dagenais G, Pogue J, et al. Vitamin E supplementation and cardiovascular events in high-risk patients. The Heart Outcomes Prevention
Evaluation Study Investigators. N Engl J Med 2000;342:154-60.

5. Mathur P, Ding Z, Saldeen T, et al. Tocopherols in the prevention and treatment of atherosclerosis and related cardiovascular disease. Clin Cardiol 2015;38:570-76.

6. Saldeen T, Li D, Mehta JL. Differential effects of alpha- and gammatocopherol on low-density lipoprotein oxidation, superoxide activity, platelet aggregation and arterial thrombogenesis. J Am Coll Cardiol 1999;34:1208-15.

7. Mehta JL, Chen J, Hermonat PL, et al. Lectin-like, oxidized low-density lipoprotein receptor-1 (LOX-1): A critical player in the development of atherosclerosis and related disorders. Cardiovasc Res 2006;69:36-45. 